\title{
ENRAIZAMIENTO DE ESTACAS DE Pinus patula
}

\author{
ROOTING OF Pinus patula CUTTINGS
}

\section{Moisés O. Rivera-Rodríguez ${ }^{1}$, J. Jesús Vargas-Hernández ${ }^{2 *}$, Javier López-Upton², Ángel Villegas-Monter ${ }^{3}$ y Marcos Jiménez-Casas ${ }^{2}$}

\begin{abstract}
${ }^{1}$ Comisión Nacional Forestal, Periférico Poniente No. 5360, Col. San Juan de Ocotán. 45019, Zapopan, Jal. ²Posgrado en Ciencias Forestales, ${ }^{3} P o s-$ grado Recursos Genéticos y Productividad - Fruticultura, Colegio de Postgraduados. Km 36.5 Carr. México-Texcoco. 56230, Montecillo, Texcoco, Estado de México.
\end{abstract}

*Autor para correspondencia (vargashj@colpos.mx)

\section{RESUMEN}

Pinus patula Schiede ex Schltdl. et Cham. es un pino endémico de México, ampliamente utilizado en plantaciones comerciales en varios países. La propagación masiva de materiales mejorados de esta especie mediante el enraizado de estacas, proporciona la oportunidad para aumentar y uniformar su productividad. Se comparó el sustrato (perlita vs. mezcla de turba con vermiculita), edad de planta madre $(12,18$ y 24 meses) y dosis de ácido indolbutírico (AIB) (0, 5000 y 10000 ppm) (i.e., $\left.\mathrm{mg} \mathrm{L}^{-1}\right)$ en el enraizado de estacas de $P$. patula, mediante un arreglo factorial en diseño experimental de bloques completos al azar. La supervivencia, enraizado, número y longitud de raíces, presencia de raíces secundarias y desarrollo de callo se evaluaron después de 14 semanas. En el sustrato con perlita se presentó el mayor porcentaje de enraizado de las estacas (21.4 vs. $14.7 \%$ ) y fue evidente el efecto de la dosis de AIB y edad de planta madre en la respuesta de las estacas. La capacidad de enraizado se redujo casi a la mitad (de 29.2 a $15.8 \%$ en el sustrato de perlita) al aumentar de 12 a 24 meses la edad de las plantas madre. El AIB inhibió la formación de raíces en las estacas de menor edad, pero no en las de 24 meses. El control de los factores evaluados permitió obtener más de $50 \%$ de enraizado en uno de los tratamientos, valor aceptable en un programa operativo de clonación.

Palabras clave: Pinus patula, enraizado, estaca, sustrato, edad de plantas madre, AlB

\section{SUMMARY}

Pinus patula Schiede ex Schltdl. et Cham. is an endemic pine in México, broadly used for commercial plantings in several countries. Massive propagation of improved individuals of this species through rooted cuttings provides the opportunity to increase and homogenize its productivity. The effect of substrate (perlite vs. mix of peat-moss with vermiculite), donor plant age (12, 18 and 24 months of age) and indole butyric acid (IBA) dose (0,5000 and 10000 ppm) (i.e., $\left.\mathrm{mg} \mathrm{L}^{-1}\right)$ on rooting of $P$. patula cuttings was evaluated using a factorial layout in a complete random blocks experimental design. Survival, rooting, number and length of primary roots, presence of secondary roots and callus development were evaluated after 14 weeks. Rooting percentage (21.4 vs. $14.7 \%$ ), as well as IBA dose and donor plant age effects on cuttings response were higher in the perlite substrate. Rooting capacity was reduced almost in half (29.2 to $15.8 \%)$ when donor plant age increased from 12 to 24 months. IBA inhibited rooting in the youngest cuttings, but not in the oldest ones. Control of factors evaluated allowed to reach over $50 \%$ of rooted cuttings in one treatment combination, an acceptable value for an operational cloning program.

Index words: Pinus patula, rooting, cutting, substrate, age of donor plant, IBA.

\section{INTRODUCCIÓN}

Pinus patula Schiede ex Schltdl. et Cham. es una especie endémica de México (Farjon et al., 1997; Perry, 1991), que es considerada como una de las coníferas mexicanas más importantes debido a su abundancia, productividad y calidad de los bosques, cuyos árboles son de fuste recto, libres de nudos y buena calidad de la madera (Velázquez et al., 2004). Recientemente se han iniciado programas de mejoramiento genético con ella, mediante la selección fenotípica de individuos superiores y el establecimiento de ensayos de progenie para su evaluación genética (SalayaDomínguez et al., 2012; Valencia-Manzo y Vargas-Hernández, 2001). Con la información generada en los ensayos, se han identificado los mejores progenitores para un programa de cruzas controladas. Una opción para aumentar los beneficios de esas cruzas es la clonación masiva de las plantas obtenidas, por medio del enraizado de estacas (Bettinger et al., 2009).

La propagación por estacas consiste en separar de la planta deseada una parte del tallo, raíz u hoja y colocarla en condiciones que favorecen la formación de raíces y brotes, para producir así una nueva planta que sea idéntica genéticamente al individuo del cual procede (Hartmann et al., 2002). La propagación clonal masiva permite maximizar el rendimiento, así como la calidad y uniformidad de las plantaciones forestales comerciales (Bettinger et al., 2009; Burdon et al., 2008; Trueman y Rodger, 2006), además de obtener plantas resistentes o tolerantes a plagas y enfermedades y con mejores características morfológicas y fisiológicas (Poupard et al., 1994).

La clonación masiva de especies forestales, en particular coníferas, es difícil por los procesos de maduración ontogénica en especies leñosas, en donde la capacidad de enraizado disminuye con la edad de la planta (Amri et al., 2010; Poupard et al., 1994). En estas especies se sugiere utilizar 
plantas madre menores de tres años de edad para tener éxito en el enraizado (Mitchell y Jones, 2006; Sánchez-Zabala et al., 2008). Nelson (2003) recomienda usar plantas madre juveniles y sanas, con podas frecuentes para aumentar el número de estacas. Hamann (1998), Anderson et al. (1999) y Mitchell et al. (2004a) sugieren hacer las podas a $20 \mathrm{~cm}$ de altura o menos para estimular la producción de rebrotes juveniles.

La edad de la estaca también es importante en el enraizado. Majada et al. (2011) encontraron que en Pinus pinaster Aiton, la capacidad de enraizado disminuye con la edad del esqueje (después de la poda). La sección de la planta donde se obtiene la estaca también es importante, ya que en plantas juveniles de pinos se logra mayor enraizado con estacas apicales que con basales (Sánchez-Zabala et al., 2008).

A diferencia de los géneros Populus y Salix que se propagan fácilmente por estacas, el enraizado de estacas en coníferas y otras leñosas se favorece con auxinas, en particular ácido indolbutírico (AIB) o ácido indolacético (AIA) (Henrique et al., 2006; Hunt et al., 2011; Ruiz-García et al., 2005), aunque no en todas se requieren (Trueman y Rodger 2006). Por ello es importante definir la concentración adecuada de auxinas para enraizar especies forestales. Bielenin (2003) utilizó concentraciones de 0, 0.1, 0.3, 0.6, y $0.9 \%$ de AIB en Juniperus y Thuja, y encontró que las concentraciones intermedias (0.3 y $0.6 \%$ ) aumentan el porcentaje de enraizado. Como medio de enraizamiento se pueden utilizar diferentes tipos de sustrato (arena, turba de musgo, vermiculita y perlita, entre otros), de manera individual o en mezclas, para asegurar condiciones adecuadas de humedad, aireación, drenaje y temperatura (Hartmann et al., 2002).

En el presente trabajo se comparó el enraizado de estacas de Pinus patula al variar la edad de planta madre, la dosis de AIB y el sustrato, con el propósito de establecer un protocolo de enraizamiento para un programa de plantaciones clonales de la especie.

\section{MATERIALES Y MÉTODOS}

\section{Producción de plantas madre}

El presente trabajo se realizó en un vivero ubicado en Montecillo, Texcoco, Estado de México (19² 27' 38.25" N y $98^{\circ} 54^{\prime} 23.91^{\prime \prime}$ ) ), a $2240 \mathrm{~m}$ de altitud. Para producir las plantas madre se utilizó un lote de semilla de P. patula de Aquixtla, Puebla (19 43' 11.9” N y $97^{\circ} 59^{\prime} 21.3^{\prime \prime}$ O, a 2930 $\mathrm{m})$. Se hicieron tres siembras escalonadas con seis meses de diferencia entre ellas para generar plantas madre de di- ferentes edades: el 13 de agosto de 2011, el 10 de febrero de 2012 y el 13 de agosto de 2012. El ensayo de enraizado inició el 12 de agosto de 2013, cuando las plantas madre tenían 24, 18 y 12 meses, respectivamente, a partir de su establecimiento.

La semilla se sembró en contenedores de $180 \mathrm{~cm}^{3}$ Ilenados con sustrato esterilizado, conformado por corteza de pino composteada, turba, perlita y vermiculita $(4: 2: 1: 1 \mathrm{v} / \mathrm{v})$, al cual se agregaron $4 \mathrm{~kg} \mathrm{~m}^{-3}$ de fertilizante de liberación controlada (18N-06P-12K+Me) por 8 meses. Semanalmente se aplicaron fungicidas (tiabendazol $2 \mathrm{~g} \mathrm{~L}^{-1} \mathrm{y}$ propamocarb $2 \mathrm{~mL} \mathrm{~L}^{-1}$ ) e insecticidas (imidacloprid y deltametrina 2 $\mathrm{mL} \mathrm{L}^{-1}$ ) como preventivos para evitar problemas de plagas o enfermedades. A los 6 meses de la siembra, las plantas de cada grupo se trasplantaron a macetas individuales de $8 \mathrm{dm}^{3}$ para iniciar la formación de setos, en un sustrato de aserrín composteado, tierra de monte, perlita y vermiculita $(4: 2: 1: 1 \mathrm{v} / \mathrm{v})$, con $4 \mathrm{~kg} \mathrm{~m}^{-3}$ del mismo fertilizante de liberación controlada (18N-06P-12K+Me) de 8 meses, y una capa de tezontle en el fondo para mejorar el drenaje.

Las plantas se podaron de manera recurrente a partir de los seis meses para estimular la formación de rebrotes. La primera poda se realizó a una altura de 10 a $12 \mathrm{~cm}, 3 \mathrm{~d}$ antes del trasplante a las macetas, y después cada vez que los rebrotes alcanzaban de 12 a $15 \mathrm{~cm}$ de longitud. Cada grupo de plantas tenía diferente tamaño y cantidad de rebrotes al momento de iniciar el estacado. Con el propósito de reducir la variabilidad en vigor de los brotes se eliminaron los débiles de cada planta.

\section{Establecimiento del ensayo}

Se utilizó una cámara de enraizado de 2.7 m de largo, 1.2 $\mathrm{m}$ de ancho y $70 \mathrm{~cm}$ de alto (fabricación propia), con sistema de nebulización controlado para mantener la humedad relativa arriba de $85 \%$ y un régimen térmico noche/día entre $17 \pm 2$ y $24 \pm 2{ }^{\circ} \mathrm{C}$. Los sustratos utilizados fueron perlita $\left(S_{1}\right)$ y una mezcla de vermiculita y turba 4:1 $\mathrm{v} / \mathrm{v}\left(\mathrm{S}_{2}\right)$, ambos previamente esterilizados. Antes de colocar las estacas se aplicó un riego a los sustratos para humedecerlos y evitar la deshidratación de las estacas; en todos los riegos durante el ensayo se utilizó agua destilada grado 2.

A las plantas madre se les aplicó fungicida (propamocarb $1.5 \mathrm{~mL} \mathrm{~L}^{-1}$ ) e insecticida (deltametrina $1.5 \mathrm{~mL} \mathrm{~L}^{-1}$ ) un día antes de cortar las estacas de 8 a $10 \mathrm{~cm}$ de longitud. Una vez cortadas se sumergieron en agua y cloro comercial $(0.25$ $\mathrm{mL} \mathrm{L}^{-1}$ ) durante 5 min y después en fungicida (propamocarb $1 \mathrm{~mL} \mathrm{~L}^{-1}$ ). Las hojas de los primeros $3 \mathrm{~cm}$ arriba del corte basal se eliminaron para introducir esta sección en el sustrato. 
El ácido indolbutírico (AIB) se aplicó impregnando la parte basal de la estaca con Radix ${ }^{\circledR}$ (producto comercial en polvo); se utilizaron concentraciones de 0, 5000 y 10,000 ppm (i.e., $\mathrm{mg} \mathrm{L}^{-1}$ ). Cada estaca se introdujo en un orificio de $3 \mathrm{~cm}$ de profundidad realizado en el sustrato. Después de establecidas, se aplicaron fungicidas e insecticidas preventivos en forma rotativa.

\section{Diseño experimental}

Se utilizó un arreglo factorial $2 \times 3 \times 3$ en un diseño experimental de bloques completos al azar con cuatro repeticiones. El factor "sustrato" con dos niveles $\left(S_{1}\right.$ y $S_{2}$ ) se consideró como ambiente de enraizado y los bloques completos se anidaron en cada uno de ellos; la combinación de los factores "edad de planta madre" con tres niveles (12, 18 y 24 meses) y "dosis de AIB" con tres niveles (0, 5000 y 10,000 ppm) se asignaron al azar a las parcelas en cada bloque. La unidad experimental fue de 10 estacas.

\section{Variables evaluadas}

La evaluación se hizo a las 14 semanas de establecido el experimento. Las variables de respuesta fueron supervivencia, número de estacas con raíces, número y longitud de raíces por estaca, presencia de raíces secundarias y estacas con callo. La estaca se consideró muerta cuando tenía necrosada más de $50 \%$ de su longitud, y enraizada cuando tenía al menos una raíz igual o mayor de $1 \mathrm{~mm}$ de largo. En cada estaca enraizada se contó el número de raíces primarias con longitud igual o mayor a $1 \mathrm{~mm}$, se midió la longitud de la raíz más larga y se registró la presencia/ ausencia de raíces secundarias. En las estacas vivas que no enraizaron se registró la presencia/ausencia de callo (abultamiento por división celular en la parte de la herida), y con esos datos se calculó el porcentaje de estacas con callo con respecto al total de estacas no enraizadas.

\section{Análisis estadístico de los datos}

En el análisis estadístico se utilizaron los valores promedio por unidad experimental con el procedimiento GLM del paquete estadístico SAS, versión 9.3 (SAS, 2000). Las variables con distribución binomial (supervivencia, estacas con raíces, presencia de raíces secundarias y estacas con callo) fueron transformadas antes del análisis con la función arco seno de la raíz cuadrada del valor original (expresado en fracción decimal). El modelo de análisis de varianza utilizado fue el siguiente:

$$
Y_{i j k l}=\mu+S_{i}+R_{j(i)}+D_{k}+E_{l}+S D_{i k}+S E_{i l}+D E_{k l}+S D E_{i k l}+\varepsilon_{i j k l}
$$

donde: $Y_{i j k l}$ es el valor de la variable observada en el j-ésimo bloque del $i$-ésimo sustrato, con la k-ésima dosis de AIB y la
I-ésima edad de planta madre; $\mu$ es la media general; $S_{j}$ es el efecto fijo del $i$-ésimo sustrato; $R_{j(i)}$ es el efecto aleatorio del $j$-ésimo bloque anidado en el $i$-ésimo sustrato; $D_{k}$ es el efecto fijo de la $k$-ésima dosis de AIB; $E_{\text {l }}$ es el efecto de la I-ésima edad de planta madre; $S D_{i k}$ es el efecto fijo de la interacción del $i$-ésimo sustrato con la k-ésima dosis de AlB; $S E_{i i}$ es el efecto fijo de la interacción del $i$-ésimo sustrato y la l-ésima edad de planta madre; $D E_{k l}$ es el efecto fijo de la interacción de la $k$-ésima dosis de AlB y la l-ésima edad de planta madre; $S D E_{i k l}$ es el efecto fijo de la interacción del $i$-ésimo sustrato, $k$-ésima dosis de AlB y l-ésima edad; y $\varepsilon_{i j k l}$ es el error experimental.

En las variables con efecto significativo de algún factor se realizó la comparación de medias con la prueba de Tukey con $\mathrm{P}=0.10$ para determinar las diferencias entre los niveles de dichos factores. Dado que en el análisis de varianza se encontraron diferencias significativas entre sustratos y en su interacción con los otros factores, se hizo un segundo análisis con un modelo reducido para evaluar el efecto de los otros factores en cada sustrato por separado.

\section{RESULTADOS Y DISCUSIÓN}

\section{Análisis de varianza}

En el análisis global se encontraron diferencias significativas entre sustratos en el enraizado $(P \leq 0.05)$, así como en la supervivencia, longitud de raíz y presencia de callo, P $\leq$ 0.10 (Cuadro 1). El porcentaje de enraizado también mostró diferencias significativas $(P \leq 0.05)$, debido a la edad de planta madre y a la interacción dosis $\times$ sustrato. La dosis de ácido indolbutírico (AIB) aplicado y la interacción dosis $\times$ edad arrojaron una $P \leq 0.10$. La interacción de los tres factores mostró efectos significativos en la supervivencia $(P \leq 0.05)$, así como en el enraizado y presencia de callo ( $P$ $\leq 0.10)$.

\section{Efecto del sustrato}

En el sustrato $\mathrm{S}_{2}$ (mezcla de vermiculita y turba) se obtuvo mayor supervivencia (94.4 vs. $90.3 \%$ ), longitud promedio de raíz y porcentaje de callo en estacas no enraizadas, que en $\mathrm{S}_{1}$ (perlita), pero en éste se obtuvo mayor enraizado de estacas, 21.4 vs. $14.7 \%$ (Cuadro 2). Estudios anteriores muestran que la vermiculita y turba tienen mayor capacidad de retención de agua y menor porosidad que la perlita (Cabrera, 1999), lo cual influye en la capacidad de enraizado de las estacas de especies leñosas (Rein et al., 1991). El efecto se atribuye a que el exceso de humedad reduce el oxígeno e inhibe la formación de iniciales de raíz, o estimula la presencia de hongos patógenos (Loach, 1985; Murthy y Goldfarb, 2001). 
Cuadro 1. Análisis de varianza de las variables de enraizamiento de las estacas de Pinus patula en el análisis conjunto.

\begin{tabular}{lcccccc}
\hline Fuente de variación & Supervivencia (\%) & Enraizado (\%) & RP (Núm.) & LR (cm) & PRS (\%) & PC (\%) \\
\hline Sustrato (Sust) & 0.098 & 0.042 & $n s$ & 0.059 & NS & 0.079 \\
Dosis & $n s$ & 0.062 & $n s$ & $n s$ & $n s$ & $n s$ \\
Edad & $n s$ & 0.028 & $n s$ & $n s$ & $n s$ & $n s$ \\
Dosis $\times$ Sust & $n s$ & 0.002 & $n s$ & $n s$ & $n s$ & $n s$ \\
Edad $\times$ Sust & $n s$ & $n s$ & $n s$ & $n s$ & $n s$ & $n s$ \\
Dosis $\times$ Edad & $n s$ & 0.057 & $n s$ & $n s$ & $n s$ & $n s$ \\
Dosis $\times$ Edad $\times$ Sust & 0.047 & 0.068 & $n s$ & $n s$ & $n s$ & 0.076 \\
\hline
\end{tabular}

RP: raíces primarias; LR: Iongitud de raíz; PRS: presencia de raíces secundarias; PC: presencia de callo en estacas no enraizadas, ns: sin diferencias significativas con $\mathrm{P}=0.10$.

Sin embargo, en este ensayo con P. patula la supervivencia de estacas fue alta en los dos sustratos y no se detectó la presencia de patógenos, por lo que el principal efecto del exceso de humedad fue la inhibición de diferenciación de raíces. Otros estudios señalan que para obtener resultados óptimos de enraizado se requiere el estímulo de un estrés hídrico ligero a moderado, como en Pinus taeda L. (Greenwood et al., 1980; Lebude et al., 2004).

Se han reportado resultados favorables de la perlita como sustrato en el enraizado de estacas de P. pinaster (Majada et al., 2011), híbridos de P. elliottii Engelm. var. elliottii $\times$ P. caribaea var. hondurensis W. H. Barrett et Golfari (Hunt et al., 2011), Juniperus scopulorum Sarg. y Thuja occidentalis L. (Bielenin, 2003), y Taxodium distichum (L.) Rich (King et al., 2011); sin embargo, en Pinus leiophylla Schiede ex Schltdl. et Cham. el uso de perlita no aumentó el porcentaje de enraizado con respecto al obtenido con la mezcla de turba, perlita y vermiculita 1:1:1 v/v (Cuevas-Cruz et al., 2015).

El análisis de varianza para cada sustrato confirmó los resultados con el modelo completo en cuanto al efecto del sustrato y su interacción con los otros factores sobre la capacidad de enraizado de las estacas. Únicamente en $\mathrm{S}_{1}$ (perlita) se encontraron efectos significativos de la dosis de AlB, edad de planta madre, y su interacción, en el porcentaje de enraizado de las estacas (Cuadro 3). En $\mathrm{S}_{2}$ se encontró efecto significativo de la interacción dosis de AIB $\times$ edad de planta madre en la supervivencia y formación de callo, pero en las otras variables no se detectaron efectos significativos de los factores ni de su interacción (Cuadro 3).

\section{Efecto de la dosis de auxina}

En el sustrato $\mathrm{S}_{2}$ el AIB no tuvo efecto significativo en la capacidad de enraizado, pero en $\mathrm{S}_{1}$ (perlita) la auxina exógena inhibió la formación de raíces, ya que se obtuvo 35.8 $\%$ de enraizado cuando no se aplicó AIB, 2.5 veces más que con la aplicación de 5000 o 10,000 ppm (Cuadro 4). Las dosis de AIB utilizadas no afectaron las otras características (supervivencia, número de raíces primarias, longitud de raíz, presencia de raíces secundarias y presencia de callo) en las estacas (Cuadro 4).

De forma natural, las auxinas vegetales intervienen en la formación de raíces en estacas (Cuisance, 1988). El uso de auxinas es importante en especies que enraizan con dificultad (Hartmann et al., 2002). Sin embargo, el problema es determinar en qué especies se requiere y cuál es la dosis adecuada. Los resultados de este estudio coinciden con información previa en Pinus patula (Aparicio-Rentería et al., 2014; Mitchell et al., 2004b) y en otras coníferas como P. pinaster (Majada et al., 2011), P. taeda (Alcantara et al., 2008), Juniperus procera Hochst. ex Endl. (Nagash, 2002), y Taxodium distichum (King et al., 2011), en donde se ha encontrado que no es indispensable aplicar auxinas para estimular el enraizado de estacas juveniles; en estos casos se obtuvieron porcentajes de enraizado similares, o incluso mayores, sin aplicar auxinas. En nuestros resultados no se encontraron efectos significativos del AIB en cuanto al número y calidad de las raíces formadas, a pesar de que hay estudios que mencionan un efecto positivo en estas características (Alcantara et al., 2008; King et al., 2011; Majada et

Cuadro 2. Valores medios ( \pm error estándar) por tipo de sustrato de las variables evaluadas en el ensayo de enraizado de estacas de Pinus patula.

\begin{tabular}{lcccccc}
\hline Sustrato & Supervivencia (\%) & Enraizado (\%) & RP (Núm.) & LR (cm) & PRS (\%) & PC (\%) \\
\hline Perlita & $90.3 \pm 2.1 \mathrm{~b}$ & $21.4 \pm 3.7 \mathrm{a}$ & $1.2 \pm 0.2 \mathrm{a}$ & $3.4 \pm 0.5 \mathrm{~b}$ & $25.8 \pm 6.5 \mathrm{a}$ & $87.7 \pm 2.9 \mathrm{~b}$ \\
Verm. + turba & $94.4 \pm 1.5 \mathrm{a}$ & $14.7 \pm 2.1 \mathrm{~b}$ & $1.4 \pm 0.2 \mathrm{a}$ & $4.5 \pm 0.6 \mathrm{a}$ & $19.4 \pm 6.4 \mathrm{a}$ & $93.4 \pm 1.7 \mathrm{a}$ \\
\hline
\end{tabular}

RP: raíces primarias; LR: longitud de raíz; PRS: presencia de raíces secundarias; PC: presencia de callo en estacas no enraizadas. Medias en una columna con letras iguales no son estadísticamente diferentes (Tukey, 0.10). 
Cuadro 3. Análisis de varianza de las variables de enraizamiento de las estacas de Pinus patula en el análisis por separado para cada sustrato.

\begin{tabular}{|c|c|c|c|c|c|c|}
\hline Fuente de variación & Supervivencia (\%) & Enraizado (\%) & RP (Núm.) & LR (cm) & PRS (\%) & PC (\%) \\
\hline \multicolumn{7}{|c|}{ Sustrato 1 (perlita) } \\
\hline Dosis & ns & 0.001 & ns & ns & ns & ns \\
\hline Edad & ns & 0.069 & ns & ns & ns & ns \\
\hline Dosis $\times$ Edad & ns & 0.009 & ns & ns & ns & ns \\
\hline \multicolumn{7}{|c|}{ Sustrato 2 (vermiculita + turba) } \\
\hline Dosis & ns & ns & ns & ns & ns & ns \\
\hline Edad & ns & ns & ns & ns & ns & ns \\
\hline Dosis $\times$ Edad & 0.025 & ns & ns & ns & ns & 0.017 \\
\hline
\end{tabular}

RP: raíces primarias; LR: Iongitud de raíz; PRS: presencia de raíces secundarias; PC: presencia de callo en estacas no enraizadas; ns: sin diferencias significativas con $\mathrm{P}=0.10$.

Cuadro 4. Valores medios ( \pm error estándar) por dosis de AIB en cada tipo de sustrato, de las variables evaluadas en el ensayo de enraizamiento de estacas de Pinus patula.

\begin{tabular}{lcccccc}
\hline Dosis AIB (ppm) & Supervivencia (\%) & Enraizado (\%) & RP (Núm.) & LR (cm) & PRS (\%) & PC (\%) \\
\hline \multicolumn{7}{c}{ Sustrato 1 (perlita) } \\
0 & $90.8 \pm 4.3 \mathrm{a}$ & $35.8 \pm 7.6 \mathrm{a}$ & $1.3 \pm 0.2 \mathrm{a}$ & $3.6 \pm 0.8 \mathrm{a}$ & $21.5 \pm 8.4 \mathrm{a}$ & $86.7 \pm 7.1 \mathrm{a}$ \\
5000 & $92.5 \pm 2.7 \mathrm{a}$ & $14.2 \pm 5.1 \mathrm{~b}$ & $1.0 \pm 0.3 \mathrm{a}$ & $3.4 \pm 1.0 \mathrm{a}$ & $26.2 \pm 14.4 \mathrm{a}$ & $90.9 \pm 2.7 \mathrm{a}$ \\
10,000 & $87.5 \pm 4.1 \mathrm{a}$ & $14.2 \pm 4.5 \mathrm{~b}$ & $1.3 \pm 0.4 \mathrm{a}$ & $3.2 \pm 1.2 \mathrm{a}$ & $32.1 \pm 14.1 \mathrm{a}$ & $85.4 \pm 4.6 \mathrm{a}$ \\
0 & $94.2 \pm 3.3 \mathrm{a}$ & $10.8 \pm 2.6 \mathrm{a}$ & $1.4 \pm 0.4 \mathrm{a}$ & $3.3 \pm 1.0 \mathrm{a}$ & $33.3 \pm 16.6 \mathrm{a}$ & $93.9 \pm 3.5 \mathrm{a}$ \\
5000 & $94.2 \pm 1.9 \mathrm{a}$ & $19.2 \pm 5.0 \mathrm{a}$ & $1.4 \pm 0.3 \mathrm{a}$ & $4.5 \pm 0.8 \mathrm{a}$ & $5.8 \pm 3.9 \mathrm{a}$ & $91.9 \pm 2.6 \mathrm{a}$ \\
10,000 & $95.0 \pm 2.8 \mathrm{a}$ & $14.2 \pm 2.9 \mathrm{a}$ & $1.2 \pm 0.2 \mathrm{a}$ & $5.7 \pm 1.1 \mathrm{a}$ & $20.5 \pm 10.0 \mathrm{a}$ & $94.4 \pm 3.2 \mathrm{a}$ \\
\hline
\end{tabular}

RP: raíces primarias; LR: longitud de raíz; PRS: presencia de raíces secundarias; PC: presencia de callo en estacas no enraizadas. Medias en una columna con letras iguales, dentro del mismo sustrato, no son estadísticamente diferentes (Tukey, 0.10).

al., 2011; Navarrete-Luna y Vargas-Hernández, 2005).

\section{Efecto de la edad de planta madre}

La edad de planta madre tuvo efecto similar en la capacidad de enraizado en los dos sustratos (Cuadro 1), con reducción gradual en el porcentaje de enraizado conforme aumenta la edad (Cuadro 5). Sin embargo, las diferencias fueron significativas únicamente en el sustrato $S_{1}$ en el que las estacas de plantas de un año alcanzaron casi el doble del enraizado (29.2 \%) que las de dos años (Cuadro 5). Al igual que la dosis de ácido indolbutírico (AIB), la edad de planta madre no afectó ninguna de las otras variables en las estacas.

Los resultados obtenidos sugieren que la capacidad de enraizamiento se reduce rápidamente conforme aumenta la edad ontogénica de la planta madre, a pesar de que se utilicen podas frecuentes para mantener su juvenilidad. Esta tendencia coincide con los datos en Pinus patula (Mitchell et al., 2004b), P. taeda (Hamman, 1998), P. radiata D.
Don (Sánchez-Zabala et al., 2008), P. pinaster (Majada et al., 2011) Juniperus procera (Nagash, 2002), Larix decidua Mill. (Peer y Greenwood, 2001) y Dalbergia melanoxylon Guill. \& Perr. (Amri et al., 2010), entre otras especies, aunque la edad de maduración y la pérdida en capacidad de enraizado difieren ampliamente entre ellas. Hackett (1985), Poupard et al. (1994) y Amri et al. (2010) señalaron que en las especies leñosas, la madurez ontogénica de la planta madre es un factor importante en la capacidad de enraizado de estacas, debido a efectos epigenéticos relacionados con cambios en la expresión génica, a medida que la planta envejece (Mitchell et al. 2004a, 2004b). Los resultados obtenidos en nuestro estudio muestran que estos cambios ontogénicos ocurren a temprana edad en Pinus patula.

\section{Interacción de la dosis de AIB con la edad de planta madre}

La interacción "dosis de AIB" × "edad de planta madre" también tuvo efecto significativo sobre el enraizamiento de las estacas en el sustrato $S_{1}$ y en la supervivencia de 
estacas y presencia de callo en $S_{2}$ (Figura 1). En el primer caso, las estacas obtenidas de plantas con 12 meses de edad mostraron mayor capacidad de enraizado (57.5\%) sin aplicar AIB, mientras que en las estacas de plantas con 24 meses se notó la tendencia a aumentar el porcentaje de enraizado con 10,000 ppm de AIB, aunque las diferencias con respecto al testigo no fueron significativas (Figura 1A). En cambio, en $\mathrm{S}_{2}$ la combinación más favorable para el enraizado (27.5\%) se obtuvo con plantas de 12 meses y 5000 ppm de AlB (Figura 1B).

La interacción significativa entre dosis de AIB y edad de planta madre en la supervivencia y formación de callo detectada en $\mathrm{S}_{2}$ se asoció con un comportamiento distinto de las estacas obtenidas de plantas madre de 18 meses a la dosis de AIB, con respecto a las estacas de plantas de 12 y 24 meses de edad (Figura 1D y1F).

En estudios con especies leñosas (como Juniperus procera, Gmelina arborea Roxb., Taxus globosa Schltdl. y Pinus pinaster) se ha reportado que la dosis óptima de auxinas para promover el enraizado varía en función de diversos factores, como la juvenilidad y estado fisiológico de la planta donante, la edad, tipo y región de la estaca en la planta o la época del año en que se obtienen (Majada et al., 2011; Muñoz-Gutiérrez et al., 2009; Nagash, 2002; Ruiz-García et al., 2005). En Pinus patula, las estacas obtenidas de material juvenil hasta de 12 meses, no requieren aplicación de auxinas y se inhibe la formación de raíces al aumentar la dosis aplicada, mientras que en estacas de plantas de mayor edad el AIB estimula la formación de raíces.

A pesar del bajo porcentaje de enraizado de estacas de Pinus patula que se obtuvo de manera global en el ensayo, los resultados muestran que una combinación de los diferentes factores evaluados permitió obtener casi $60 \%$ de estacas enraizadas en tres meses, valor cercano al prome- dio que se ha obtenido en otros estudios con esta especie (Aparicio-Rentería et al., 2014; Mitchell et al., 2004b), lo cual resulta aceptable en un programa operativo de clonación. Además, existe la posibilidad de aumentar esta capacidad de enraizado si se controlan otros factores de establecimiento y manejo de setos y estacas.

Es posible que la escasa respuesta al enraizado en la mayoría de los tratamientos se deba a la época del año en que se estableció el ensayo y a las condiciones de temperatura, ya que en la cámara de enraizado solo fue posible controlar las temperaturas altas (mayores a $25^{\circ} \mathrm{C}$ ), pero no las bajas (menores a $17^{\circ} \mathrm{C}$ ), y durante el ensayo se presentaron eventos de bajas temperaturas $\left(-4.9\right.$ a $14^{\circ} \mathrm{C}$ a intemperie), generalmente durante las primeras horas del día. Hartmann et al. (2002) mencionan que el éxito en el enraizamiento depende de la época del año, lo que está relacionado tanto con el estado fisiológico de las plantas, como con las condiciones ambientales durante el enraizado.

\section{CONCLUSIONES}

Pinus patula es difícil de enraizar, por la maduración relativamente acelerada que reduce su capacidad para inducir raíces en estacas después del primer año de edad; las podas no permitieron alargar el periodo de juvenilidad. La aplicación de auxinas exógenas inhibe la capacidad de enraizado en material juvenil de esta especie, pero en estacas de plantas de 24 meses no se manifiesta dicho efecto, por lo que su uso en dosis de 5000 y 10,000 ppm podría contrarrestar el efecto de maduración de los setos. La utilización de la perlita como sustrato, con menor capacidad de retención de humedad y mayor aireación que la mezcla de vermiculita y turba, tuvo efecto positivo en el enraizado de las estacas de P. patula. Si se controlan de manera adecuada los factores anteriores es posible asegurar un enraizamiento cercano a $60 \%$, valor aceptable en un

Cuadro 5. Valores medios ( \pm error estándar) por edad de planta madre en cada tipo de sustrato, de las variables evaluadas en el ensayo de enraizamiento de estacas de Pinus patula.

\begin{tabular}{lcccccc}
\hline Edad (meses) & Supervivencia (\%) & Enraizado (\%) & RP (Núm.) & LR (cm) & PRS (\%) & PC (\%) \\
\hline \multicolumn{7}{c}{ Sustrato 1 (perlita) } \\
12 & $87.5 \pm 4.9 \mathrm{a}$ & $29.2 \pm 8.2 \mathrm{a}$ & $1.4 \pm 0.3 \mathrm{a}$ & $3.8 \pm 1.2 \mathrm{a}$ & $36.7 \pm 13.5 \mathrm{a}$ & $83.6 \pm 7.3 \mathrm{a}$ \\
18 & $87.5 \pm 3.7 \mathrm{a}$ & $19.2 \pm 6.5 \mathrm{ab}$ & $1.1 \pm 0.3 \mathrm{a}$ & $3.7 \pm 1.1 \mathrm{a}$ & $24.1 \pm 13.2 \mathrm{a}$ & $85.6 \pm 4.2 \mathrm{a}$ \\
24 & $95.8 \pm 1.5 \mathrm{a}$ & $15.8 \pm 3.7 \mathrm{~b}$ & $1.1 \pm 0.2 \mathrm{a}$ & $2.7 \pm 0.7 \mathrm{a}$ & $17.6 \pm 7.4 \mathrm{a}$ & $93.9 \pm 1.8 \mathrm{a}$ \\
12 & $95.0 \pm 2.3 \mathrm{a}$ & $19.2 \pm 5.3 \mathrm{a}$ & $1.8 \pm 0.4 \mathrm{a}$ & $4.5 \pm 1.1 \mathrm{a}$ & $33.3 \pm 12.4 \mathrm{a}$ & $93.0 \pm 3.0 \mathrm{a}$ \\
18 & $91.7 \pm 3.8 \mathrm{a}$ & $13.3 \pm 2.6 \mathrm{a}$ & $1.2 \pm 0.2 \mathrm{a}$ & $5.8 \pm 0.9 \mathrm{a}$ & $18.2 \pm 12.2 \mathrm{a}$ & $91.0 \pm 4.1 \mathrm{a}$ \\
24 & $96.7 \pm 1.4 \mathrm{a}$ & $11.7 \pm 2.4 \mathrm{a}$ & $1.1 \pm 0.3 \mathrm{a}$ & $3.2 \pm 0.9 \mathrm{~b}$ & $5.6 \pm 5.6 \mathrm{a}$ & $96.2 \pm 1.6 \mathrm{a}$ \\
\hline
\end{tabular}

RP: raíces primarias; LR: longitud de raíz; PRS: presencia de raíces secundarias; PC: presencia de callo en estacas no enraizadas. Medias en una columna con letras iguales, dentro del mismo sustrato, no son estadísticamente diferentes (Tukey, 0.10). 

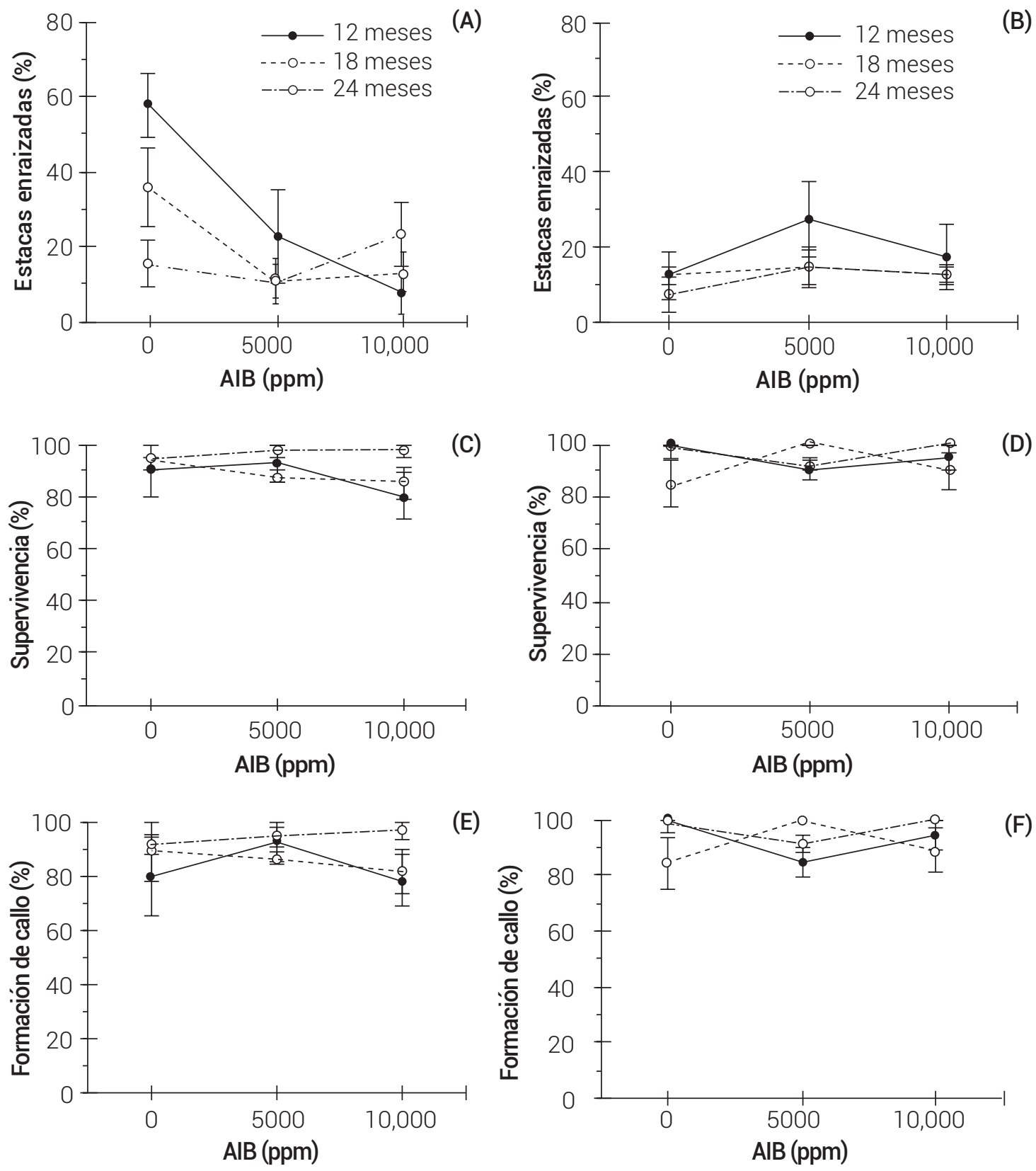

(F)

Figura 1. Enraizado $(A, B)$, supervivencia (C, D) y formación de callo $(E, F)$ en estacas de Pinus patula evaluadas en sustrato conformado por perlita (A, C, E) y la mezcla de vermiculita y turba (B, D, F), en función de la edad de planta madre y dosis de AIB.

programa de propagación (clonación) de la especie a partir de materiales seleccionados.

\section{AGRADECIMIENTOS}

Al Ing. León Jorge Castaños-Martínez e Ing. Salvador Castro Zavala, propietarios de la "Reserva Forestal Multifuncional" en Aquixtla, Puebla, el lote de semillas proporcionado para la realización de este trabajo. También agrade- cemos los comentarios proporcionados por los revisores y el editor asignados por la revista, que contribuyeron a mejorar el contenido del manuscrito.

\section{BIBLIOGRAFÍA}

Alcantara G. B., L. Lopes F. R., A. Rioyei H. and K. C. Zufellato R. (2008) Effect of indolbutyric acid (IBA) and the collection of shoots in different seasons of the year on minicutting rooting of Pinus taeda $\mathrm{L}$. Scientia Forestalis Piracicaba 36:151-156.

Amri E., H. V. M. Lyaruu, A. S. Nyomora and Z. L. Kanyeka (2010) Vegetative propagation of African Blackwood (Dalbergia melanoxylon 
Guill. \& Perr.): effects of age of donor plant, IBA treatment and cutting position on rooting ability of stem cuttings. New Forests 39:183-194.

Anderson A. B., L. J. Frampton and R. J. Weir (1999) Shoot production and rooting ability of cuttings from juvenile greenhouse loblolly pine hedges. Transactions of the Illinois State Academy of Science 92:1-14.

Aparicio-Rentería A., S. F. Juárez-Cerrillo y L. R. Sánchez-Velásquez (2014) Propagación por enraizamiento de estacas y conservación de árboles plus extintos de Pinus patula procedentes del norte de Veracruz, México. Madera y Bosques 20:85-96.

Bettinger P., M. Clutter, J. Siry, M. Kane and J. Pait (2009) Broad implications of southern United States pine clonal forestry on planning and management of forests. International Forestry Review 11:331-345.

Bielenin M. (2003) Rooting and gas exchange of conifer cuttings treated with indolbutyric acid. Journal of Fruit and Ornamental Plant Research 11:99-105

Burdon R. D., M. J. Carson and C. J. A. Shelbourne (2008) Achievements in forest tree genetic improvement in Australia and New Zealand. 10. Pinus radiata in New Zealand. Australian Forestry 71:263279

Cabrera R. I. (1999) Propiedades, uso y manejo de sustratos de cultivo para la producción de plantas en maceta. Revista Chapingo Serie Horticultura 5:5-11.

Cuevas-Cruz J. C., M. Jiménez-Casas, J. Jasso-Mata, P. Pérez-Rodríguez, J. López-Upton and A. Villegas-Monter (2015) Asexual propagation of Pinus leiophylla Schiede ex Schltdl. et Cham. Revista Chapingo Serie Ciencias Forestales y del Ambiente 21:81-95.

Cuisance P. (1988) La Multiplicación de las Plantas y el Vivero. Ed. Mundi-Prensa. Madrid, España. 165 p.

Farjon A., J. A. Pérez de la Rosa and B. T. Styles (1997) Field Guide to the Pines of Mexico and Central America. Royal Botanic Gardens, Kew, UK. $147 \mathrm{p}$

Greenwood M. S., T. M. Marino, R. D. Meier and K. W. Shahan (1980) The role of mist and chemical treatments in rooting loblolly and shortleaf pine cuttings. Forest Science 26:651-655.

Hackett W. P. (1985) Juvenility, maturation, and rejuvenation in woody plants. In: Horticultural Reviews, volume 7. J. Janick (ed.). John Wiley \& Sons, Inc., Hoboken, NJ, USA. pp:109-155.

Hamann A. (1998) Adventitious root formation in cuttings of loblolly pine (Pinus taeda L.): developmental sequence and effects of maturation. Trees Structure and Function 12:175-180.

Hartmann H. T., D. E. Kester, F. T. Davies Jr. and R. L. Geneve (2002) Plant Propagation, Principles and Practices. 7th ed. Prentice-Hall, Upper Saddle River, New Jersey. 880 p.

Henrique A., E. Nogueira C., E. Orika O. and S. Zambello de P. (2006) Effect of plant growth regulators in the rooting of Pinus cuttings. Brazilian Archives of Biology and Technology 49:189-196.

Hunt M. A., S. J. Trueman and A. Rasmussen (2011) Indole-3-butyric acid accelerates adventitious root formation and impedes shoot growth of Pinus elliottii var. elliottii and P. caribaea var. hondurensis cuttings. New Forests 41:349-360.

King A. R., M. A. Arnold, D. F. Welsh and W. T. Watson (2011) Substrates, wounding, and growth regulator concentrations alter adventitious rooting of Baldcypress cuttings. HortScience 46:13871393.

Lebude A. V., B. Goldfarb, F. A. Blazich, F. C. Wise and J. Frampton (2004) Mist, substrate water potential and cutting water potential influence rooting of stem cuttings of loblolly pine. Tree Physiology 24:823-831.

Loach K. (1985) Rooting of cuttings in relation to the propagation medium. Combined Proceedings International Plant Propagators'
Society 35:472-485.

Majada J., C. Martínez-Alonso, I. Feito, A. Kidelman, I. Aranda and R. Alia (2011) Mini-cuttings: an effective technique for the propagation of Pinus pinaster Ait. New Forests 41:399-412.

Mitchell R. G. and N. B. Jones (2006) The effects of ontogenetic maturation in Pinus patula-Part II: field performance. Southern African Forestry Journal 207:3-9.

Mitchell R. G., J. Zwolinski and N. B. Jones (2004a) A review on the effects of donor maturation on rooting and field performance of conifer cuttings. Southern African Forestry Journal 201:53-63.

Mitchell R. G., J. Zwolinski and N. B. Jones (2004b) The effects of ontogenetic maturation in Pinus patula - Part 1: nursery performance. Southern African Forestry Journal 202:29-36.

Murthy R. and B. Goldfarb (2001) Effect of handling and water stress on water status and rooting of loblolly pine stem cuttings. New Forests 21:217-230

Muñoz-Gutiérrez L., J. J. Vargas-Hernández, J. López-Upton and M. SotoHernández (2009) Effect of cutting age and substrate temperature on rooting of Taxus globosa. New Forests 38:187-196.

Nagash L. (2002) Successful vegetative propagation techniques for the threatened African pencil cedar (Juniperus procera Hoechst. ex Endl.). Forest Ecology and Management 161:53-64.

Navarrete-Luna M. y J. J. Vargas-Hernández (2005) Propagación asexual de clones de Eucalyptus camaldulensis Dehnh, utilizando radix en diferentes concentraciones. Revista Chapingo Serie Ciencias Forestales y del Ambiente 11:111-116.

Nelson W. (2003) Propagating Plantation Trees from Cuttings in Containers. Management Ltd. Christchurch, New Zeland. $18 \mathrm{p}$.

Peer K. R. and M. S. Greenwood (2001) Maturation, topophysis and other factors in relation to rooting in Larix. Tree Physiology 21:267272.

Perry J. P. (1991) The Pines of Mexico and Central America. Ed. Timber Press. Portland, Oregon, USA. $231 \mathrm{p}$

Poupard C., M. Chauviere and O. Monteuuis (1994) Rooting Acacia mangium cuttings: effects of age, within shoot position and auxin treatment. Silvae Genetica 43:226-231.

Rein W. H., R. D. Wright and J. R. Seiler (1991) Propagation medium moisture level influences adventitious rooting of woody stem cuttings. Journal of the American Society for Horticultural Science 116:632-636

Ruiz-García R., J. J. Vargas-Hernández, V. M. Cetina-Alcalá y A. Villegas-Monter (2005) Efecto del ácido indolbutírico (AIB) y tipo de estaca en el enraizado de Gmelina arborea Roxb. Revista Fitotecnia Mexicana 28:319-326.

Salaya-Domínguez J. M., J. López-Upton y J. J. Vargas-Hernández (2012) Variación genética y ambiental en dos ensayos de progenies de Pinus patula. Agrociencia 46:519-534.

Sánchez-Zabala J., U. Ortega-Lasuen, J. Majad-Guijo, K. Txarterina-Urkiri y M. Duñabeitia-Aurrekoetxea (2008) Optimización de la propagación vegetativa por estaquillado de genotipos de interés comercial de Pinus radiata. Cuadernos de la Sociedad Española de Ciencias Forestales 28:201-205.

SAS Institute (2000) The SAS system for Windows. Release 9.3. SAS Inst., Cary, NC. USA.

Trueman S. J. and F. P. Rodger (2006) Propagation of Wollemi pine from tip cuttings and lower segment cuttings does not require rooting hormones. Scientia Horticulturae 109:394-397.

Valencia-Manzo S. y J. J. Vargas-Hernández (2001) Correlaciones genéticas y selección simultánea del crecimiento y densidad de la madera de Pinus patula. Agrociencia 35:109-119.

Velázquez M. A., G. Ángeles P., T. Llanderal 0., A. R. Román J. y J. V. Reyes H. (2004) Monografía de Pinus patula. SEMARNAT/CONAFOR. Colegio de Postgraduados. Zapopan, Jal. 124 p. 